\title{
Le rôle de l'université dans le développement de son milieu
}

\author{
François Paquette ${ }^{1}$ \\ Ministère de l'Éducation, du Loisir et du Sport, \\ Saguenay-Lac-Saint-Jean
}

Cet article résume les propos tenus lors d'une table ronde animée par M. Marc-Urbain Proulx (Université du Québec à Chicoutimi) réunissant MM. Gilles Bergeron (Université du Québec à Chicoutimi), Guy Massicotte (Université du Québec) et Paul Prévost (Université de Sherbrooke) tenue dans le cadre du colloque Inégalités sociales et recomposition des territoires : de la mesure à l'intervention du Congrès de l'ACFAS 2005.

A vec la création en 1968 de l'Université du Québec qui s’ajoutait au réseau universitaire déjà établi, la société québécoise s’est donnée les moyens d'étendre l'accès aux études universitaires dans l'ensemble des régions et de favoriser l'émergence d'axes de développement liés aux caractéristiques et aux besoins des zones d'appartenance des universités.

On connaît relativement bien les impacts économiques générés par la présence des universités dans leurs milieux locaux et régionaux. Il n'en est cependant pas de même lorsqu'il est question de jauger l'importance de leur apport au développement de ces milieux. Les recherches menées dans leurs enceintes et la circulation de plus en plus rapide des connaissances habilitent les universités à jouer un rôle significatif dans l'émergence et l'accroissement de la capacité d'innover locale et régionale. Dans quelle mesure jouent-elles ce rôle et par quels moyens le font-elles ? À l'intérieur d'une dynamique de développement des collectivités, quels rapports les universités entretiennent-elles avec les intervenants socio-économiques de leurs milieux ?

D’entrée de jeu, M. Guy Massicotte précise qu’il faut saisir combien le contexte dans lequel les universités évoluent a changé au cours des dernières décennies pour comprendre le type de rapports que les universités entretiennent aujourd'hui avec leurs milieux. On constate d'abord que la demande du marché pour des diplômés dépasse le volume de l'offre. Cette situation amène les universités à chercher à augmenter leurs effectifs étudiants, notamment en facilitant l'accès à la formation universitaire.

Les universités désirent également se rapprocher des bassins de clientèles potentielles. Alors que, traditionnellement, on concevait l'accès à la formation universitaire en fonction de la situation géographique des institutions, on remarque que, depuis vingt ans, les universités dispensent de plus en plus de programmes en dehors de leurs campus et même dans plusieurs régions ainsi qu’à l'étranger. Cette délocalisation de l'enseignement universitaire, alliée à l'accroissement des activités d'enseignement à distance, modifie considérablement la notion de territoire d'influence des universités, ce qui peut amener les intervenants locaux et régionaux à s'interroger parfois sur l'appartenance des institutions à leur milieu.

Les universités québécoises sont appelées à agir partout dans le monde. Elles développent des créneaux de spécialisation scientifique afin de se positionner avantageusement sur la scène internationale. Toutefois, cet éclatement de la dimension territoriale du rayonnement universitaire ne signifie pas que les universités sont moins disponibles pour participer au développement de leur milieu immédiat. En effet, le prolongement de leurs forces scientifiques au plan global ne peut se réaliser que dans la mesure où ces forces prennent leur source dans une implication régionale.

On assiste parallèlement à un télescopage du temps dans les champs de l'enseignement et de la recherche. 
Les technologies de l'information et de la communication ont permis une accélération phénoménale de la production de connaissances. Le développement du savoir se fait maintenant en temps réel, ce qui entraîne une rapide obsolescence des acquis scientifiques et l'obligation, pour les chercheurs, de s'inscrire dans un processus de formation continue.

Les lieux de production des connaissances se sont multipliés dans le monde, et les universités ne détiennent plus de monopole dans ce domaine. Les institutions qui veulent soutenir la concurrence, en particulier au regard du maintien d'une expertise de pointe et de l'accès aux sources de financement de la recherche, en plus de se spécialiser, doivent développer des réseaux scientifiques d'échange et de partage tant dans la communauté universitaire qu'à l'extérieur de celle-ci. D'ailleurs, ce mode de fonctionnement en réseaux est adopté par tous les intervenants, que ce soit les professeurs, les étudiants ou les acteurs socioéconomiques avec lesquels les universités transigent.

On remarque par ailleurs que les universités tirent plusieurs avantages de leur partenariat avec le milieu. Le déplacement de l'axe de production des connaissances amène les chercheurs universitaires à faire certains apprentissages dans les milieux de pratique mêmes. On peut dire, à cet égard, que l'université apprend autant du milieu que celui-ci de l'université.

Pour M. Gilles Bergeron, il ne fait aucun doute que les universités participent activement au développement de leur milieu et qu'elles le font dans le respect des trois volets de leur mission : l'enseignement, la recherche et les services à la collectivité. Ainsi, au regard de ce dernier aspect, les universités collaborent avec leur milieu pour trouver des utilisations innovatrices de leurs infrastructures : il s'agit alors de leur donner une plus grande valeur ajoutée en diversifiant leur utilisation pour répondre aux besoins générés par les dynamiques socioculturelles du milieu.

Il va de soi que les activités de formation contribuent à doter le milieu de ressources humaines qualifiées dans de multiples domaines. L'accessibilité à la formation de base et à la formation continue revêt une importance appréciable pour le renforcement du milieu socio-économique local et régional, entre autres par l'accroissement de la rétention des diplômés dans leur milieu et par l'intégration de nouveaux arrivants, des étudiants et des professeurs notamment, phénomène en hausse actuellement dans les régions.
Au plan de la recherche, l'élaboration de thématiques proches des besoins du milieu est primordiale et, à ce chapitre, la réalisation d'activités de transfert en partenariat avec les acteurs du milieu est de première importance. On a peu évalué les effets de ces activités sur le développement local et régional et on peut s'interroger sur la nature de leurs impacts. M. Bergeron convient que des études restent à faire sur cette question.

\section{La collaboration avec le milieu n'est pas une pratique naturelle dans les universités.}

Prenant appui sur l'histoire récente du partenariat réunissant l'université et le milieu dans le domaine de l'aluminium au Saguenay-Lac-Saint-Jean, M. Bergeron fait remarquer que des activités de transfert ont été porteuses de résultats concrets. Elles se sont déroulées sur trois scènes spécifiques: d'abord dans le milieu industriel même, alors que des connaissances, des techniques et des processus industriels nouveaux ont été développés grâce à des partenariats entre la grande entreprise et des chercheurs universitaires. Ensuite, on a vu apparaître des petites et moyennes entreprises qui ont mis en application des éléments de ce corpus d'innovations. Enfin, on a assisté à l'apparition d'innovations chez les sous-traitants qui peuvent ainsi soutenir le rythme du développement de l'industrie.

S'il ne remet pas en question l'intérêt et l'importance du partenariat, M. Paul Prévost fait remarquer cependant que la collaboration avec les universités n'est pas aisée pour les intervenants du milieu. Plusieurs problèmes et mésententes surviennent dans les relations entre les partenaires à cause de la friction de cultures organisationnelles différentes. Alors que les acteurs locaux et régionaux conçoivent la réalisation de projets dans une optique entrepreneuriale qui privilégie l'action et l'obtention rapide de résultats, l'université fonctionne dans un univers institutionnel complexe et relativement fermé sur lui-même.

La collaboration avec le milieu n'est pas une pratique naturelle dans les universités. Celles-ci s'y sont engagées graduellement, au fur et à mesure qu'elles éprouvaient des besoins financiers grandissants. La recherche de partenaires visait alors à trouver de nouvelles 
ressources qui leur permettraient de continuer à se développer. Les universités doivent maintenant opérer un changement de cap significatif dans le contexte du développement de partenariats avec le milieu puisque leur collaboration doit être envisagée dans une perspective de réponse équitable aux attentes et aux objectifs de tous les partenaires impliqués.

\section{L'université n'a pas encore trouvé les moyens de valoriser les services à la collectivité à l'intérieur de ses modes de fonctionnement.}

Trois systèmes spécifiques sont imbriqués pour constituer l'organisation universitaire : les systèmes politique, bureaucratique et entrepreneurial. Le premier est formé des multiples instances décisionnelles et des rapports qu'elles entretiennent entre elles. Le second est constitué par l'appareil administratif chargé de mettre en œuvre les décisions et d'assurer l'intégrité des processus administratifs mis en place à cet effet. Le troisième système, moins formel, est composé de professeurs engagés dans des activités de recherche qui sont en quête de partenaires et de financement pour la réalisation de leurs projets.

M. Prévost remarque que les acteurs du milieu ne comprennent pas cette complexité organisationnelle. Ils ne savent pas à qui s'adresser ni comment procéder pour proposer des projets conjoints aux universités. Ils redoutent la multiplicité des structures et des intervenants ainsi que la lenteur des processus décisionnels et administratifs. Selon lui, la façon la plus efficace de faire agir l'université est d'intéresser en premier lieu les entrepreneurs universitaires. Ceux-ci voudront s'impliquer dans la mesure où les projets proposés coïncident avec leurs intérêts professionnels. Si des professeurs considèrent que l'université devrait collaborer à la réalisation des projets, ils feront le nécessaire pour amener le système politique à y consacrer les ressources suffisantes. Cette implication de l'université devra finalement être sanctionnée par des ententes formelles qui satisferont aux exigences bureaucratiques.

L'université n'a pas encore trouvé les moyens de valoriser les services à la collectivité à l'intérieur de ses modes de fonctionnement. Ainsi, la progression de carrière des professeurs est largement tributaire de leurs travaux de recherche, mais les activités de transfert ne sont pas reconnues au même titre, ce qui a pour effet de limiter l'intérêt ou la disponibilité des professeurs pour des projets en partenariat. Les universités devraient élaborer et mettre en place des mécanismes organisationnels et financiers qui permettent le développement concomitant de la recherche traditionnelle, de la recherche-action et des activités de transfert.

Par ailleurs, le contrôle bureaucratique exercé dans les universités n'est pas adapté à la gestion de projet qui a cours dans le milieu. Il est conçu pour satisfaire aux exigences des fonds de recherche ou pour correspondre aux pratiques de départements comme ceux de sciences physiques qui ont la capacité de générer des revenus. Il n'a cependant pas la souplesse requise pour s'adapter au rythme de réalisation de projets influencés par l'évolution de la conjoncture.

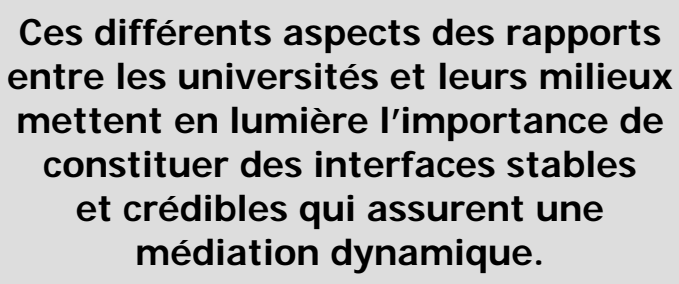

Les activités de transfert peuvent jouer un rôle appréciable dans la formation des étudiants. En effet, elles constituent autant d'opportunités de travail concret sur le terrain, ce qui contribue à rehausser la pertinence et la qualité des apprentissages des étudiants. Cette mise en contact avec les acteurs du milieu contribue également à retenir les étudiants en région après l'obtention de leur diplôme en leur donnant la possibilité de commencer à développer leur réseau professionnel pendant leur formation.

Ces différents aspects des rapports entre les universités et leurs milieux mettent en lumière l'importance de constituer des interfaces stables et crédibles qui assurent une médiation dynamique. D’ailleurs, $\mathrm{M}$. Massicotte remarque que les universités accordent une importance grandissante à l'innovation sociale, c'està-dire à la médiation entre les créateurs de savoir et les utilisateurs de ces connaissances. Elles déploient de plus en plus d'énergie pour mettre en place des mécanismes qui facilitent l'accès aux ressources universitaires (Services à la collectivité de l'UQAM) ou pour créer des lieux de concertation et de coopération 
sectoriels (Consortium de recherche sur la forêt boréale commerciale de l'UQAC; Institut des sciences de la mer de Rimouski) qui stimulent les échanges impliquant chercheurs et praticiens dans des processus itératifs de construction de savoirs.

Tous conviennent finalement que le partenariat entre l'université et son milieu doit faire l'objet d'une certaine vigilance éthique. L'université, comme partenaire à part entière, doit apporter la rigueur méthodologique nécessaire, stimuler la conceptualisation et maintenir un certain esprit critique. Cette distance critique est nécessaire afin de garantir l'intégrité et la qualité des résultats obtenus et permettre aux partenaires de réviser périodiquement leurs moyens et leurs actions en regard des objectifs poursuivis.
L’université a-t-elle un rôle à jouer dans le développement local et régional ? Les participants à la table ronde en sont convaincus. Ce rôle comporte de multiples aspects qui appellent la collaboration et la participation de l'ensemble de l'organisation universitaire afin que celle-ci puisse agir de façon plurielle dans tous les secteurs de la vie sociale, économique et culturelle de son milieu.

\section{Note}

1 François Paquette a œuvré dans plusieurs régions du Québec au cours de sa carrière. Il est actuellement directeur régional du MELS au Saguenay-Lac-Saint-Jean. Il est détenteur d'une maîtrise en histoire de l'art de l'Université de Montréal et d'une maîtrise en administration publique de l'École nationale d'administration publique. 